Progress in Flight Physics 5 (2013) 441-456

DOI: $10.1051 /$ eucass/201305441

(C) Owned by the authors, published by EDP Sciences, 2013

\title{
NUMERICAL INVESTIGATION OF TWO-PHASE FLOW STRUCTURE AND HEAT TRANSFER IN A SUPERSONIC DUSTY GAS FLOW OVER A BLUNT BODY
}

\author{
B. Oesterle ${ }^{1}$, A. N. Volkov ${ }^{2}$, and Yu. M. Tsirkunov ${ }^{3}$ \\ ${ }^{1}$ LEMTA UMR CNRS 7563, ESSTIN \\ Henri Poincare University \\ Nancy, France \\ ${ }^{2}$ Department of Materials Science and Engineering \\ University of Virginia \\ Charlottesville, USA \\ ${ }^{3}$ Faculty of Aerospace Engineering \\ Baltic State Technical University \\ St. Petersburg, Russia
}

\begin{abstract}
A dusty gas flow near the forward part of a blunt body is investigated numerically by an example of a crosswise supersonic flow over a cylinder. Considerable attention is paid to the fine flow structure and the temperature field of the carrier gas inside the boundary layer. Wide ranges of particle sizes and free stream particle volume fraction have been studied, and the possible interparticle collisions and two-way coupling effects have been taken into account. A combined computational fluid dynamics (CFD) / Monte Carlo method has been used for the computational simulation of the two-phase flow. The heat flux from the carrier gas and the energy flux from the particles to the body surface have been calculated. It has been found that the heat flux depends nonmonotonically on the particle size. The maximum heat flux has been observed for particles whose radius is just greater than the so-called critical particle radius, which is defined as the radius above which the particles reach the body surface and collide with it (particles with a radius below the critical one do not reach the surface). A physical explanation is proposed for this phenomenon which seems unexpected at first glance. The effects of the contact particle-wall heat transfer and the turbulence generation by particles are estimated.
\end{abstract}




\section{INTRODUCTION}

As is well known from available experiments, the presence of dispersed solid particles in a high speed gas flow over a body can increase essentially the heat transfer near the stagnation point. Originally, this was revealed for coarse-grained particles impinging on the body surface with high velocity [1]. In this case, the transformation of the particle kinetic energy into heat at the surface yields a crucial contribution to the total heat flux near the stagnation point. Numerous phenomenological models of the heat transfer at the stagnation region were proposed for this case (see, e.g. [2-4]). For fine particles which do not reach the body surface, the effect of a considerable heat flux increase at the blunt body surface was first predicted theoretically [5] and much later this phenomenon was revealed by experiments [6]. It is obvious that the gas flow field modification by particles near the body is the single reason of the heat transfer enhancement in this case. The theoretical prediction [5] was based on the two-way coupling flow model inside the boundary layer, whereas the reverse effect of particles on the gas flow outside the boundary layer was not taken into account. In the majority of theoretical studies, including the above cited papers, the interparticle collisions were not taken into account. Apparently, the single exception is the paper [7] in which a Monte Carlo simulation of the collisional "gas" of particles is applied to study the wall heat transfer by impinging a two-phase jet. However, the theoretical estimates show that the collisions between incident and rebounded coarse-grained particles in the gas flow over a blunt body begin to play an essential role at much less particle concentration in the free-stream than that at which the reverse effect of the dispersed phase on the carrier gas flow field becomes important (see, e.g., [8]). A direct computational simulation of gas-particle flow over a cylinder confirmed completely these estimates [9, 10]. If particles are so fine that they do not collide with the body surface, the two-way coupling effects become essential not only inside the boundary layer, but also outside it at very low free-stream particle concentration (at $\alpha_{\mathrm{p} \infty} \sim 10^{-5}$ ) [11].

In the present study, the flow model [10] is used which takes into account the following important effects: $(i)$ the boundary layer on the body surface; $(i i)$ the interparticle collisions; and (iii) the two-way coupling effects. This model is described briefly in section 2 .

The main objective of the study is to investigate the influence of the particle phase on the carrier gas flow field in the stagnation region as well as on the heat flux from the carrier gas and the energy flux from the dispersed phase to the body surface in wide ranges of particle sizes and free-stream particle volume fractions. The flow regimes with and without interparticle collisions are considered. Particular attention is given to the particles whose radius is in the vicinity of the critical value $r_{\mathrm{p} *}$ which separates the particles into two groups: the particles of a radius smaller than $r_{\mathrm{p} *}$ do not reach the body surface, larger particles collide with the surface. 


\section{MATHEMATICAL MODEL OF TWO-PHASE GAS-PARTICLE FLOW}

A supersonic gas-particle flow over a blunt body at rather high Reynolds number is considered so that a thin viscous boundary layer is formed on the forward part of the body surface. However, the carrier gas flow is assumed to be laminar (in calculations we took $\operatorname{Re}_{\infty}=10^{5}$ ) and is described by the complete Navier-Stokes equations with the source terms modeling the action of the dispersed phase. The possible turbulence generation by particles is estimated additionally.

The dispersed phase is described by the generalized Boltzmann kinetic equation which takes into account the force, the torque, and the heat flux from the carrier gas on particles, as well as the inelastic and frictional interparticle collisions.

\subsection{Kinetic Model of the Collisional "Gas" of Particles}

The dispersed phase is considered as a discrete set of solid particles which move in the carrier gas and collide with each other. It is assumed that particles are spheres of the identical radius $r_{\mathrm{p}}$, and they interact with each other only through binary collisions. The state of the $i$ th particle is determined by a point $\mathbf{x}_{i}$ of the phase space which includes the particle radius-vector $\mathbf{r}_{i}$, the particle translational and rotational velocities ( $\mathbf{v}_{\mathrm{p} i}$ and $\omega_{\mathrm{p} i}$, respectively), and the particle temperature $T_{\mathrm{p} i}$, i.e., $\mathbf{x}_{i}=\left(\mathbf{r}_{i}, \mathbf{v}_{\mathrm{p} i}, \omega_{\mathrm{p} i}, T_{\mathrm{p} i}\right)$. The vector $\mathbf{x}_{i}$ is conveniently splitted into $\mathbf{r}_{i}$ and $\mathbf{y}_{i}=\left(\mathbf{v}_{\mathrm{p} i}, \omega_{\mathrm{p} i}, T_{\mathrm{p} i}\right)$. It is also assumed that the parameters of any two particles are not statistically correlated. Under these conditions, the state of a set of particles can be described in terms of the oneparticle probability density function (hereafter, the subscript 1 is used to denote a test particle) $f_{1}=f\left(\mathbf{x}_{1}, t\right)=f\left(\mathbf{r}_{1}, \mathbf{v}_{\mathrm{p} 1}, \omega_{\mathrm{p} 1}, T_{\mathrm{p} 1}, t\right)$, such that $f_{1} d \mathbf{x}_{1}=$ $f\left(\mathbf{r}_{1}, \mathbf{v}_{\mathrm{p} 1}, \omega_{\mathrm{p} 1}, T_{\mathrm{p} 1}, t\right) d \mathbf{r}_{1} d \mathbf{v}_{\mathrm{p} 1} d \omega_{\mathrm{p} 1} d T_{\mathrm{p} 1}$ is the number of particles with locations, velocities and temperature within the elementary volume $d \mathbf{r}_{1} d \mathbf{v}_{\mathrm{p} 1} d \omega_{\mathrm{p} 1} d T_{\mathrm{p} 1}$ in the vicinity of the point $\mathbf{x}_{1}=\left(\mathbf{r}_{1}, \mathbf{v}_{\mathrm{p} 1}, \omega_{\mathrm{p} 1}, T_{\mathrm{p} 1}\right)$. The particle numerical density $n$ is given by

$$
n\left(\mathbf{r}_{1}, t\right)=\int f\left(\mathbf{r}_{1}, \mathbf{y}_{1}, t\right) d \mathbf{y}_{1}
$$

The function $f_{1}$ satisfies the generalized Boltzmann kinetic equation [9]:

$$
\begin{array}{r}
\frac{\partial f_{1}}{\partial t}+\frac{\partial}{\partial \mathbf{r}_{1}}\left(\mathbf{v}_{\mathrm{p} 1} f_{1}\right)+\frac{\partial}{\partial \mathbf{v}_{\mathrm{p} 1}}\left(\frac{\mathbf{f}_{\mathrm{p} 1}}{m_{\mathrm{p}}} f_{1}\right)+\frac{\partial}{\partial \omega_{\mathrm{p} 1}}\left(\frac{\mathbf{l}_{\mathrm{p} 1}}{I_{\mathrm{p}}} f_{1}\right)+\frac{\partial}{\partial T_{\mathrm{p} 1}}\left(\frac{q_{\mathrm{p} 1}}{c_{\mathrm{p}}^{\circ} m_{\mathrm{p}}} f_{1}\right) \\
=I_{\text {coll }} .
\end{array}
$$


Here, $m_{p}$ and $I_{p}$ are the mass and momentum of inertia of a particle; and $c_{\mathrm{p}}^{\circ}$ is the specific heat capacity of the particle material. The collisional integral in the right-hand side is

$$
I_{\text {coll }}=4 r_{\mathrm{p}}^{2} \int d \mathbf{y}_{2} \int_{\mathbf{g}_{12} \cdot \mathbf{n}_{12} \leq 0}\left(\frac{f_{1}^{-} f_{2}^{-}}{J}-f_{1} f_{2}\right)\left|\mathbf{g}_{12} \cdot \mathbf{n}_{12}\right| \sin \chi_{12} d \chi_{12} d \varepsilon_{12} .
$$

The force $\mathbf{f}_{\mathrm{p} 1}=\mathbf{f}_{\mathrm{D}}+\mathbf{f}_{\mathrm{M}}\left(\mathbf{f}_{\mathrm{D}}\right.$ and $\mathbf{f}_{\mathrm{M}}$ are the particle drag force and the Magnus lift force, respectively), the torque $\mathbf{l}_{\mathrm{p} 1}$, and the total heat flux $q_{\mathrm{p} 1}$ are calculated for the test particle with the parameters $\left(\mathbf{v}_{\mathrm{p} 1}, \omega_{\mathrm{p} 1}, T_{\mathrm{p} 1}\right)$ at the point $\mathbf{r}_{1}$ of the flow; $f_{2}=f\left(\mathbf{r}_{1}, \mathbf{y}_{2}, t\right) ; f_{1}^{-}=f\left(\mathbf{r}_{1}, \mathbf{y}_{1}^{-}, t\right) ; f_{2}^{-}=f\left(\mathbf{r}_{1}, \mathbf{y}_{2}^{-}, t\right) ; \mathbf{n}_{12}$ is the unit vector directed from the center of the first particle towards the center of the second particle at the collision instant; and $\mathbf{g}_{12}=\mathbf{v}_{2}-\mathbf{v}_{1}$. The inequality $\mathbf{g}_{12} \cdot \mathbf{n}_{12} \leq 0$ is the condition of physical feasibility of a collision between the first and the second particles, and the angles $\chi_{12}$ and $\varepsilon_{12}$ specify the direction of $\mathbf{n}_{12}$ in spherical coordinates [9].

For the particle drag force, the Magnus force, the torque and the total heat flux, the usual expressions with dimensionless coefficients $C_{\mathrm{D}}, C_{\mathrm{M}}, C_{\mathrm{L}}$, and $\mathrm{Nu}$ (Nusselt number) are used. These coefficients were calculated from the formulae approximating the analytical, experimental and numerical data in wide ranges of the governing parameters of the flow around a single particle. Further details and references can be found in [10].

The parameter $J$ in the collisional integral given by Eq. (2) depends on the particle-particle collision model. The ratios of the postcollision normal and tangential relative velocities of the colliding particles' contact point to the precollision ones $\left(a_{r n}\right.$ and $a_{r t}$, respectively) are assumed to be constant. Then the following expression can be derived from the consideration of the phase volume change during a binary collision [9]: $J=a_{r n}^{2} a_{r t}^{2}$.

Let $\Phi=\Phi\left(\mathbf{x}_{i}\right)$ be a parameter of an individual particle. If a macro parameter of the dispersed phase $\langle\Phi\rangle(\mathbf{r}, t)$ at a point $\mathbf{r}$ of the physical space is defined as ensemble average value of $\Phi=\Phi\left(\mathbf{x}_{i}\right)$ in a unit volume of the gas-particle mixture, then $\langle\Phi\rangle(\mathbf{r}, t)$ can be expressed in terms of $\Phi$ and $f\left(\mathbf{x}_{1}, t\right)$ as follows:

$$
\langle\Phi\rangle(\mathbf{r}, t)=\int \Phi\left(\mathbf{r}, \mathbf{y}_{1}, t\right) f\left(\mathbf{r}, \mathbf{y}_{1}, t\right) d \mathbf{y}_{1} .
$$

For example, if $c_{\mathrm{p}}^{\circ}$ is temperature-independent, then the thermal energy of the particles in a unit volume of the mixture is defined as

$$
E_{\mathrm{p}}(\mathbf{r}, t)=\int c_{\mathrm{p}}^{\circ} m_{\mathrm{p}} T_{p 1} f\left(\mathbf{r}, \mathbf{y}_{1}, t\right) d \mathbf{y}_{1}
$$

and the average temperature of the particles at a point $\mathbf{r}$ is given by

$$
\left\langle T_{\mathrm{p}}\right\rangle=\frac{E_{\mathrm{p}}(\mathbf{r}, t)}{\int c_{\mathrm{p}}^{\circ} m_{\mathrm{p}} f\left(\mathbf{r}, \mathbf{y}_{1}, t\right) d \mathbf{y}_{1}} .
$$




\subsection{Governing Equations for the Carrier Gas Flow}

The governing equations for two-dimensional (2D) flows of the viscous and thermal conducting carrier gas can be written in the following compact form [10]:

$$
\frac{\partial \mathbf{A}}{\partial t}+\frac{\partial \mathbf{B}}{\partial x}+\frac{\partial \mathbf{C}}{\partial y}=\frac{\partial \mathbf{G}}{\partial x}+\frac{\partial \mathbf{H}}{\partial y}+\mathbf{F}
$$

where $\mathbf{A}, \mathbf{B}, \mathbf{C}, \mathbf{G}$, and $\mathbf{H}$ are the vectors defined by

$$
\begin{gathered}
\mathbf{A}=\left(\begin{array}{c}
\rho e \\
\rho \\
\rho u \\
\rho v
\end{array}\right) ; \quad \mathbf{B}=\left(\begin{array}{c}
u(\rho e+p) \\
\rho u \\
\rho u^{2}+p \\
\rho u v
\end{array}\right) ; \quad \mathbf{C}=\left(\begin{array}{c}
v(\rho e+p) \\
\rho v \\
\rho u v \\
\rho v^{2}+p
\end{array}\right) ; \\
\mathbf{G}=\left(\begin{array}{c}
u \tau_{x x}+v \tau_{x y}-q_{x} \\
0 \\
\tau_{x x} \\
\tau_{x y}
\end{array}\right) ; \quad \mathbf{H}=\left(\begin{array}{c}
u \tau_{x y}+v \tau_{y y}-q_{y} \\
0 \\
\tau_{x y} \\
\tau_{y y}
\end{array}\right) .
\end{gathered}
$$

The equation of state of the carrier gas, the relationship for the total energy, and the relationships for the components of the heat flux and the viscous stress tensor are given by

$$
\begin{gathered}
p=\rho \Re T ; \quad e=c_{v} T+\frac{u^{2}+v^{2}}{2} ; \quad q_{x}=-\kappa \frac{\partial T}{\partial x} ; \quad q_{y}=-\kappa \frac{\partial T}{\partial y} \\
\tau_{x x}=\frac{2}{3} \mu\left(2 \frac{\partial u}{\partial x}-\frac{\partial v}{\partial y}\right) ; \quad \tau_{y y}=\frac{2}{3} \mu\left(2 \frac{\partial v}{\partial y}-\frac{\partial u}{\partial x}\right) ; \quad \tau_{x y}=\mu\left(\frac{\partial u}{\partial y}+\frac{\partial v}{\partial x}\right) .
\end{gathered}
$$

The coefficients of viscosity and conductivity are calculated from the formulae $\mu=\mu_{s}\left(T / T_{s}\right)^{3 / 2}\left(T_{s}+C_{s}\right) /\left(T+C_{s}\right)$ and $\kappa=c_{\mathrm{p}} \mu / \operatorname{Pr}$. Here, $c_{v}$ and $c_{\mathrm{p}}$ are the gas heat capacity at constant volume and constant pressure, and $\operatorname{Pr}$ is the Prandtl number.

The source term $\mathbf{F}$ in Eq. (3) describes the averaged effect of the particles on the carrier gas at the point $\mathbf{r}$, and it can be expressed in terms of the function $f_{1}=f(\mathbf{r}, \mathbf{y}, t)$ as follows:

$$
\mathbf{F}(\mathbf{r}, t)=-\langle\mathbf{Q}\rangle(\mathbf{r}, t)=-\int \mathbf{Q}\left(\mathbf{r}, \mathbf{y}_{1}\right) f\left(\mathbf{r}, \mathbf{y}_{1}, t\right) d \mathbf{y}_{1} .
$$

Here, $\mathbf{Q}=\mathbf{Q}\left(\mathbf{x}_{1}\right)=\left(a_{\mathrm{p} 1}, 0, f_{\mathrm{p} 1 x}, f_{\mathrm{p} 1 y}\right)$ where $a_{\mathrm{p} 1}=\mathbf{f}_{\mathrm{p} 1} \cdot \mathbf{v}_{\mathrm{p} 1}+\mathbf{l}_{\mathrm{p} 1} \cdot \omega_{\mathrm{p} 1}+q_{\mathrm{p} 1}$ is the rate of change of the total energy of a particle due to the gas-particle interaction and $f_{\mathrm{p} 1 x}$ and $f_{\mathrm{p} 1 y}$ are the $x$ - and $y$-components of the force $\mathbf{f}_{\mathrm{p} 1}$. 


\subsection{Boundary Conditions}

A uniform supersonic crosswise dusty gas flow over a circular cylinder is considered. A sketch of the flow structure is shown in Fig. 1. The computational domain $A B C D A$ in the plane of flow $(x, y)$ is bounded by the entrance boundary $A D$ placed in the undisturbed flow upstream from the cylinder, the exit boundary $C D$ placed in the middle cross section of the cylinder, the axis of symmetry $A B$, and the body contour $B C$.

For the Navier-Stokes equations (3), the conventional boundary conditions are used at all boundaries. The formulation of the boundary conditions for the kinetic equation (1) is similar to that for the classical Boltzmann equation in the rarefied gas dynamics.

In the free stream, the particles are assumed to be nonrotating and their translational velocity and temperature are equal to those of the carrier gas $\mathbf{v}_{\infty}$ and $T_{\infty}$. In this case, the boundary condition at the entrance boundary has the form: $f\left(\mathbf{x}_{1}, t\right)=n_{\mathrm{p} \infty} \delta_{3}\left(\mathbf{v}_{\mathrm{p} 1}-\mathbf{v}_{\infty}\right) \delta_{3}\left(\omega_{\mathrm{p} 1}\right) \delta_{1}\left(T_{\mathrm{p} 1}-T_{\infty}\right)$, where $n_{\mathrm{p} \infty}$ is the particle number density in the free stream, and $\delta_{m}$ is the $m$-dimensional Dirac function.

The authors exclude particles flying off the computational domain from further calculations and assume that particles do not enter the domain through the exit boundary $C D$.

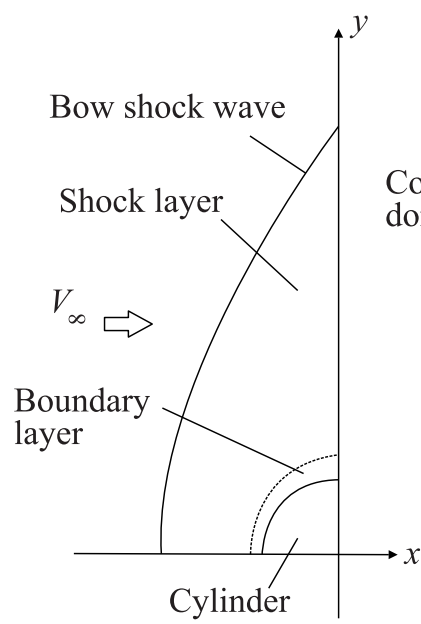

(a)

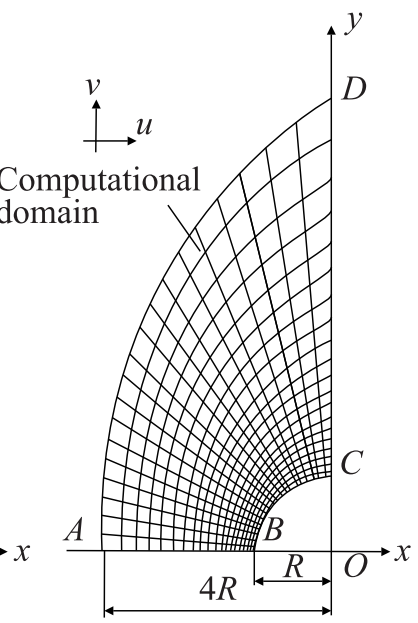

(b)

Figure 1 Sketch of $(a)$ the flow structure; and $(b)$ the grid for the carrier gas in the computational domain 
In the plane of flow symmetry, the symmetry condition is taken: $f\left(\mathbf{x}_{1}, t\right)$ $=f\left(\mathbf{r}_{1}, \mathbf{y}_{1}^{\prime}, t\right)$ with $\mathbf{v}_{\mathrm{p} 1}^{\prime}=\mathbf{v}_{\mathrm{p} 1}-2\left(\mathbf{v}_{\mathrm{p} 1} \cdot \mathbf{n}\right) \mathbf{n}, \omega_{\mathrm{p} 1}^{\prime}=\omega_{\mathrm{p} 1}-2 \mathbf{n} \times\left(\omega_{\mathrm{p} 1} \times \mathbf{n}\right)$ and $T_{\mathrm{p} 1}^{\prime}=T_{\mathrm{p} 1}$ for $\mathbf{v}_{\mathrm{p} 1} \cdot \mathbf{n}<0$, where $\mathbf{n}$ is the unit vector normal to the plane of symmetry and pointed towards the computational domain.

As to the boundary condition on the body surface, it is assumed that the process of reflection of the $i$ th particle from the body is instantaneous and deterministic, so that the postcollisional particle parameters $\mathbf{y}_{i}^{+}$are uniquely determined by the precollisional ones $\mathbf{y}_{i}^{-}$and the unit normal to the surface $\mathbf{n}_{\mathrm{w}}$ at the point of particle-wall contact $\mathbf{r}_{\mathrm{w}}: \mathbf{y}_{i}^{+}=\mathbf{y}_{\mathrm{w}}^{+}\left(\mathbf{y}_{i}^{-}, \mathbf{n}_{\mathrm{w}}\right)$. In this case, the condition for $f_{1}=f\left(x_{1}, t\right)$ takes the form:

$$
\left|\mathbf{v}_{\mathrm{p} 1} \cdot \mathbf{n}_{\mathrm{w}}\right| f\left(\mathbf{x}_{1}, t\right)=\int_{\mathbf{v}_{\mathrm{p} 1}^{\prime} \mathbf{n}_{\mathrm{w}}<0}\left|\mathbf{v}_{\mathrm{p} 1}^{\prime} \cdot \mathbf{n}_{\mathrm{w}}\right| \delta_{7}\left(\mathbf{y}_{1}-\mathbf{y}_{\mathrm{w}}^{+}\left(\mathbf{y}_{1}^{\prime}, \mathbf{n}_{\mathrm{w}}\right)\right) f\left(\mathbf{r}_{\mathrm{w}}, \mathbf{y}_{1}^{\prime}, t\right) d \mathbf{y}_{1}^{\prime}
$$

The relation $\mathbf{y}_{\mathrm{w}}^{+}\left(\mathbf{y}_{i}^{-}, \mathbf{n}_{\mathrm{w}}\right)$ is determined by the particle--wall collision model. In the present study, the model based on the conservation laws and available experimental data for the particle impact velocity up to several hundred meters per second is used (see, e.g., [9]).

\section{DISCUSSION OF NUMERICAL RESULTS AND CONCLUSIONS}

The detailed description of the whole method used in simulations of two-phase flows over bodies is given in [12]. It represents the combination of a finitedifference method for the modified Navier-Stokes equations and the Direct Simulation Monte Carlo (DSMC) method for the "gas" of particles. Two boundaryfitted grids displaced relative to each other and strongly refined near the body contour were constructed in the computational domain for the continuous and dispersed phases (the grid for the carrier gas is shown schematically in Fig. 1b). The number of grid cells was equal to 120 along the polar angle and 240 along the polar radius. The concentration of grid lines increased $10^{3}$ times towards the body contour in order to provide high accuracy of calculations of the carrier gas flow field inside the boundary layer. The number of simulated particles in the DSMC method was about $1.5 \cdot 10^{6}$. The steady-state solution for the twophase flow was obtained as the limit of the time-dependent solution when time $t$ increased.

In calculations, the carrier gas was air, the particles' material was corundum, the cylinder material was ductile steel. The physical constants of gas and particles, the free stream flow parameters, and the cylinder size used in simulations are given in Table 1. 
Table 1 Input data for calculations

\begin{tabular}{lc|lc}
\hline Parameter & Value & Parameter & Value \\
\hline $\mathfrak{R}, \mathrm{J} /(\mathrm{kg} \cdot \mathrm{K})$ & 286.9 & $R, \mathrm{~m}$ & 0.01 \\
$T_{\infty}, \mathrm{K}$ & 151.7 & $\gamma$ & 1.4 \\
$\mu_{s}, \mathrm{~kg} /(\mathrm{m} \cdot \mathrm{s})$ & $1.71 \cdot 10^{-5}$ & $\mathrm{Pr}$ & 0.76 \\
$T_{s}, \mathrm{~K}$ & 273 & $\mathrm{M}_{\infty}$ & 2 \\
$C_{s}, \mathrm{~K}$ & 117 & $\mathrm{Re}_{\infty}$ & $10^{5}$ \\
$\rho_{\mathrm{p}}^{\circ}, \mathrm{kg} / \mathrm{m}^{3}$ & 3950 & $T_{\mathrm{w}} / T_{0}$ & 0.5 \\
\hline
\end{tabular}

The Reynolds number $\mathrm{Re}_{\infty}$ is defined as follows:

$$
\operatorname{Re}_{\infty}=\frac{2 R \rho_{\infty} V_{\infty}}{\mu_{\infty}}
$$

The flow parameters in Table 1 correspond to $\rho_{\infty}=0.104 \mathrm{~kg} / \mathrm{m}^{3}, V_{\infty}$ $=494.5 \mathrm{~m} / \mathrm{s}$, and $T_{0}=273 \mathrm{~K}$. The temperature dependence of the particle material specific heat was approximated by the formula $c_{\mathrm{p}}^{\circ}\left(T_{\mathrm{p} i}\right)=\left(3.04 \cdot 10^{-6} T_{\mathrm{p} i}^{3}\right.$ $\left.-7.01 \cdot 10^{-3} T_{\mathrm{p} i}^{2}+5.54 T_{\mathrm{p} i}-341.8\right) \mathrm{J} /(\mathrm{kg} \cdot \mathrm{K})$. The particle radius was varied from 0.1 to $10 \mu \mathrm{m}$, the corresponding Stokes number $\operatorname{St}_{\infty}=(2 / 9) \operatorname{Re}_{\infty}\left(r_{\mathrm{p}} / r\right)^{2} \rho_{\mathrm{p}}^{\circ} / \rho_{\infty}$ ranged from 0.042 to 420 . The free stream particle volume fraction $\alpha_{\mathrm{p} \infty}$ was varied from $10^{-6}$ to $3 \cdot 10^{-5}$, corresponding to the particle mass concentration in the free stream from $3 \%$ to $53 \%$. The values of the coefficients $a_{r n}$ and $a_{r t}$ in the interparticle collision model were equal to 0.5 and 0.9 , respectively.

The particle phase flow field in such a dusty gas flow over a blunt body was studied in detail earlier [9-11]. Here, the main attention is paid to the particle induced modification of the carrier gas flow field and the heat transfer rate at the body surface.

\subsection{Carrier Gas Flow Structure}

The temperature field of the carrier gas is of principal interest in studies of the heat transfer. When the two-way coupling effects are essential, the flow field of the carrier gas and the particle phase flow structure closely correlate with each other. As it was shown in [9], the overall flow structures of coarse-grained and fine particles near the body differ radically from each other. The reason is that large particles rebound from the body surface and collide intensively with each other, whereas small particles do not reach the body surface and move without collisions.

The extent to which the gas flow field is affected by particles depends on the free stream particle concentration, all other governing parameters being the 


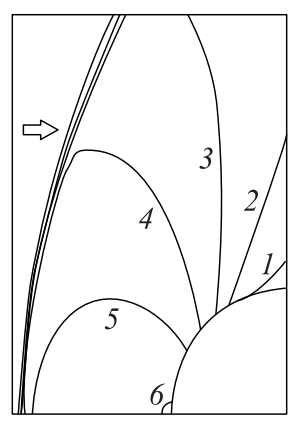

(a)

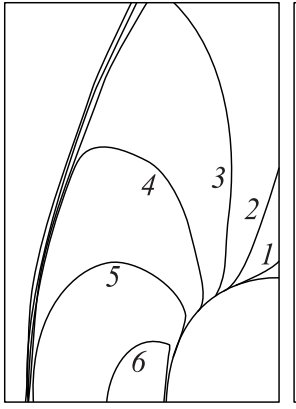

(b)

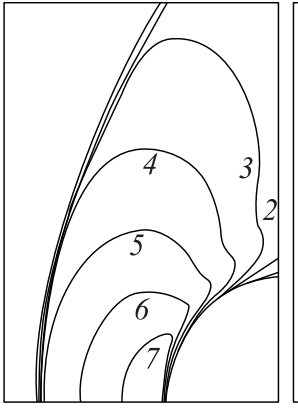

(c)

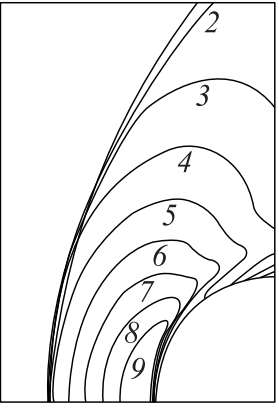

$(d)$

Figure 2 Contours of constant gas temperature: $(a) \alpha_{\mathrm{p} \infty}=0$ (dust-free gas flow); (b) $3 \cdot 10^{-6} ;(c) 10^{-5}$; and $(d) \alpha_{\mathrm{p} \infty}=3 \cdot 10^{-5}$. The curves correspond to $T / T_{\infty}=1.2(1)$, $1.4(2), 1.5(3), 1.6(4), 1.7(5), 1.8(6), 1.9(7), 2.0(8)$, and $2.1(9) ; r_{\mathrm{p}}=1 \mu \mathrm{m}$

same. Figure 2 illustrates how the gas temperature field changes as $\alpha_{\mathrm{p} \infty}$ increases. The value of $r_{\mathrm{p}}=1 \mu \mathrm{m}$ given in the caption is approximately three times larger than the critical particle radius $r_{\mathrm{p} *}$, which slightly increases with increasing $\alpha_{\mathrm{p} \infty}$. Under the flow parameters considered here, $r_{\mathrm{p} *} \approx 0.35 \mu \mathrm{m}$ (and, hence, $r_{\mathrm{p} *} / R \approx 0.35 \cdot 10^{-4}$ ) at $\alpha_{\mathrm{p} \infty}=10^{-5}$. As is seen from Fig. 2, the thickness of the shock layer decreases when $\alpha_{\mathrm{p} \infty}$ increases. Besides $\alpha_{\mathrm{p} \infty}$, this thickness depends also on the particle radius, decreasing with $r_{\mathrm{p}}$ (Fig. $3 a$ ). The gas and particle temperature distributions along the stagnation streamline (see Fig. 3) show the thermal relaxation of both phases in the flow. Outside the boundary

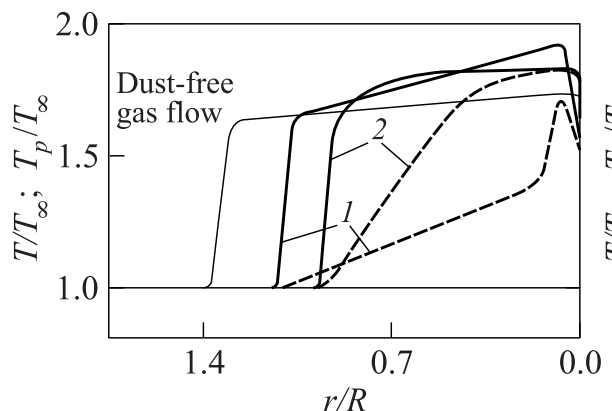

(a)

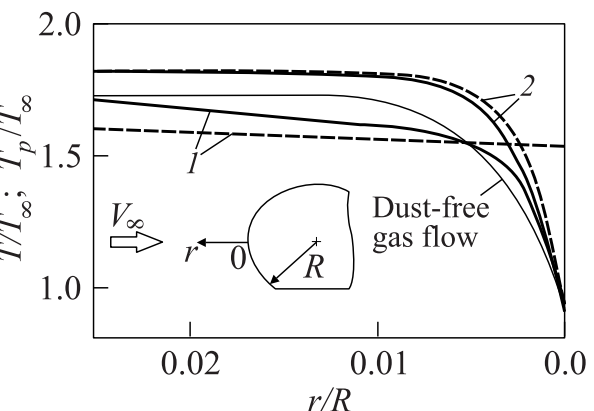

(b)

Figure 3 Distribution of gas temperature (solid curves) and dispersed phase temperature (dashed curves) along the stagnation streamline: $(a)$ in the whole shock layer; and (b) inside the boundary layer (enlarged scale): $1-r_{\mathrm{p}}=1 \mu \mathrm{m}$; and $2-r_{\mathrm{p}}=0.3 \mu \mathrm{m}$; $\alpha_{\mathrm{p} \infty}=10^{-5} ; T_{\mathrm{w}} / T_{\infty}=0.5$ 
layer, the particle temperature is always less than the gas temperature (compare the solid and dashed curves with the same numbers in Fig. 3a), meaning that the heat flux resulting from the gas-particle interaction is always directed from gas to particles. However, the presence of particles in the flow causes the gas temperature to increase. This is the result of two opposite processes: the heat exchange between gas and particles and the dissipation of the particles' kinetic energy when they decelerate in the shock layer. As is seen, the heating of the carrier gas due to the dissipation exceeds its cooling due to the heat exchange between phases. Smaller particles moving from the bow shock wave to the body are heated more rapidly (compare the dashed curves 1 and 2 in Fig. $3 a$ ). The local maximum on the dashed curve 1 in Fig. $3 a$ is caused by the thermal contribution of the reflected particles which are heated additionally when they move slowly upstream from the body surface in the hot gas flow.

In the present study, a "cold" body surface is considered $\left(T_{\mathrm{w}} / T_{0}=0.5\right.$, see Table 1). In this case, the temperature of both large and small particles inside the boundary layer near the stagnation point is found to exceed the carrier gas temperature (see the solid and dashed curves with the same numbers in Fig. $3 b$ ). In this region, the direction of the interphase heat flux changes to the opposite one, resulting in gas heating by particles near the surface, and, hence, in increasing the heat flux from the carrier gas to the body surface. It should be noted that the dissipation rate of the particles' kinetic energy in this region is negligible compared with the gas-particle heat exchange.

Figure 4 shows the profiles of tangential gas velocity $v_{\tau}$ and gas temperature $T$ in different cross sections of the boundary layer up to the polar angle $\theta=75^{\circ}$.

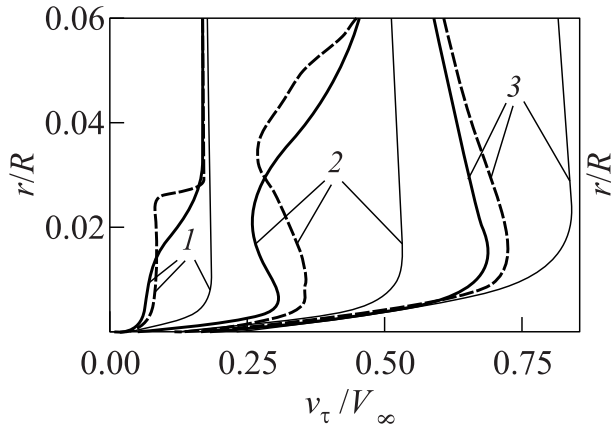

(a)

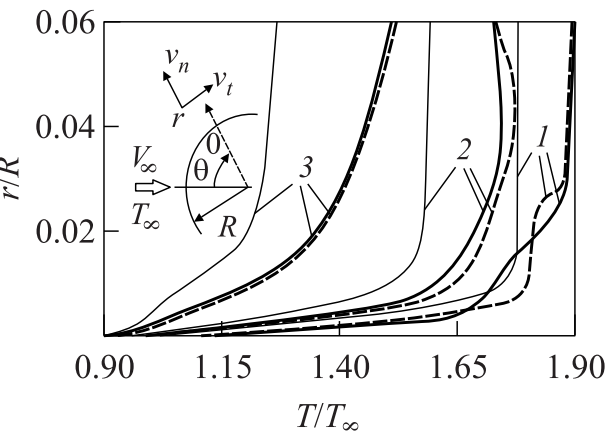

(b)

Figure 4 Profiles of gas parameters in different cross sections of the boundary layer: (a) tangential velocity; and (b) temperature; $\alpha_{\mathrm{p} \infty}=10^{-5}, r_{\mathrm{p}}=0.5 \mu \mathrm{m}$. Thin solid curves are for the dust-free gas flow; thick solid and dashed curves are for the dusty gas flow with and without interparticle collisions, respectively: $1-\theta=15^{\circ} ; 2-45^{\circ}$; and $3-\theta=75^{\circ}$ 
These profiles were calculated for the cases of the dust-free gas flow and the dusty gas flow with and without interparticle collisions. It can be seen that in the dusty gas flow with particles of the considered radius, the thickness of the boundary layer is a few time larger than in the pure gas flow. Moreover, in the vicinity of the stagnation point, the profiles in the particle laden gas flow differ qualitatively from those in the dust-free gas flow. The collisions play a very important role near the stagnation point and their effect decreases downstream along the body surface.

\subsection{Heat Flux and Energy Flux at the Body Surface}

Heating of the carrier gas by particles in the near-wall layer leads to an increase in the heat flux $q_{\mathrm{w}}=(\kappa|\partial T / \partial r|)_{\mathrm{w}}$ from the gas phase to the body surface. The heat flux at the stagnation point $q_{\mathrm{w} 0}$ vs. the particle radius $r_{\mathrm{p}}$, all other governing parameters being fixed, is shown in Fig. 5. For comparison, the same dependence is plotted for the case of the flow without interparticle collisions. The collisions enhance $q_{\mathrm{w} 0}$ in the whole range of $r_{\mathrm{p}}$ considered, with the exception of particles whose radius is less than $r_{\mathrm{p} *}$ (at the given governing parameters of the problem, $\left.r_{\mathrm{p} *} / R \approx 0.35 \cdot 10^{-4}\right)$. The ratio $q_{\mathrm{w} 0} / q_{\mathrm{w} 0}^{\circ}$ (where $q_{\mathrm{w} 0}^{\circ}$ denotes the value in the pure gas flow) depends essentially on $r_{\mathrm{p}}$. It reaches a maximum value of about 4.5 for a relative particle radius $r_{\mathrm{p}} / R=0.45 \cdot 10^{-4}$, which is slightly larger than the critical value $r_{\mathrm{p} *} / R \approx 0.35 \cdot 10^{-4}$. The latter conclusion is also true for other governing parameters of the problem, and it seems to be universal in nature. In the vicinity of the maximum, the role of interparticle collisions is particularly

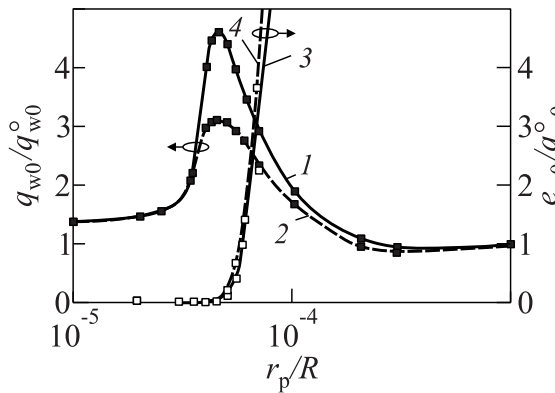

(a)

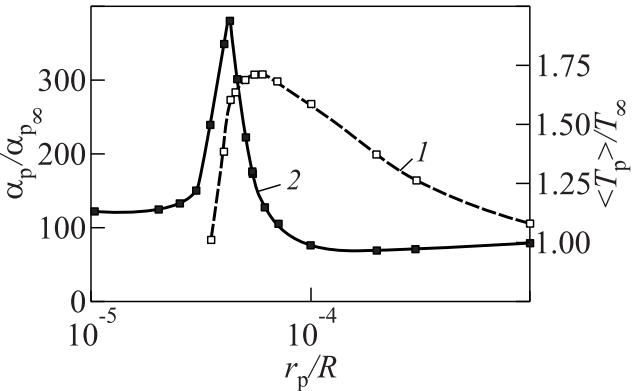

(b)

Figure 5 (a) Heat flux from the carrier gas $q_{\mathrm{w} 0}$ (curves 1 and 2) and energy flux from the dispersed phase $e_{\mathrm{w} 0}$ (curves 3 and 4 ) at the stagnation point vs. particle radius; solid and dashed curves are calculated for flows with and without interparticle collisions, respectively; and (b) particle average temperature (curve 1) and particle volume fraction (curve 2) at the stagnation point vs. particle radius; $\alpha_{\mathrm{p} \infty}=10^{-5}$; signs are the calculated points 
important. Ignoring these collisions yields a heat flux $q_{\mathrm{w} 0}$ which is about 1.5 times less than that for the collisional "gas" of particles.

In Fig. 5, the energy flux $e_{\mathrm{w} 0}$ from the dispersed phase to the surface of the cylinder at the stagnation point is also shown. This energy flux is associated with the lost of particles' kinetic energy due to particle-wall collisions which is assumed to be converted into the internal energy of the body. The magnitude of $e_{\mathrm{w} 0}$ is equal to zero for fine particles (with radii $r_{\mathrm{p}} \leq r_{\mathrm{p} *} \approx 0.35 \cdot 10^{-4} R$ ) which do not reach the body surface and, hence, do not collide with it. The energy flux $e_{\mathrm{w} 0}$ is very close to zero for slightly larger particles (with radii $0.35 \cdot 10^{-4} R$ $\leq r_{\mathrm{p}} \leq 0.45 \cdot 10^{-4} R$ ), and it increases sharply from $r_{\mathrm{p}} / R=0.45 \cdot 10^{-4}$ at which $q_{\mathrm{w} 0}$ was shown to be maximum. Thus, the total heat flux from the two-phase gas-particle flow at the stagnation point is actually determined by $q_{\mathrm{w} 0}$ if particles are rather small and by $e_{\mathrm{w} 0}$ in the case of coarse-grained particles (practically, for $\left.r_{\mathrm{p}} / R \geq 2 \cdot 10^{-4}\right)$. In the intermediate range $\left(0.45 \cdot 10^{-4} \leq r_{\mathrm{p}} / R \leq 2 \cdot 10^{-4}\right)$, both effects are important and must be taken into account. Note that the above ranges of $r_{\mathrm{p}}$ have been obtained for the considered governing parameters and these ranges will be different for other ones.

The considerable increase in $q_{\mathrm{w} 0}$ in the vicinity of $r_{\mathrm{p} *}$ is associated with the accumulation and heating of particles near the stagnation point. The particle volume fraction $\alpha_{\mathrm{p}}$ and the particle average temperature $\langle T\rangle_{\mathrm{p}}$ in the grid cell adjacent to the stagnation point are plotted in Fig. 5 as a function of the particle radius. It is seen that these curves have maxima at close but different values of $r_{\mathrm{p}}$ which, in turn, are slightly higher than $r_{\mathrm{p} *}$. The maximum of $\alpha_{\mathrm{p}}$ is sharp, whereas the maximum of $\langle T\rangle_{\mathrm{p}}$ is flat. The thermal energy of the dispersed phase is proportional to the particle mass concentration, the specific heat of the particle material, and the particle temperature. Actually, the specific heat of the particle material is a function of the particle temperature, however, it varies weakly near the maximum of $\langle T\rangle_{\mathrm{p}}$. In this case, one may suppose the specific heat to be constant for particles of different sizes in some neighborhood of the maximum. Hence, the thermal energy of the dispersed phase in this neighborhood is proportional to the product of $\alpha_{\mathrm{p}}$ and $\langle T\rangle_{\mathrm{p}}$. The maximum of $\alpha_{\mathrm{p}}\langle T\rangle_{\mathrm{p}}$ was found at $r_{\mathrm{p}} \approx 0.45 \cdot 10^{-4} R$, which is exactly the same radius at which $q_{\mathrm{w} 0}$ is maximal. Therefore, the maximum of the heat flux from the carrier gas to the body near the stagnation point is correlated with the maximum of the particle thermal energy near this point. From physical point of view, the maximal thermal energy of the dispersed phase causes the maximal heating of the carrier gas by particles and, as a result, the maximal gas temperature gradient under fixed $T_{\mathrm{w}}$ and, hence, the maximal magnitude of $q_{\mathrm{w} 0}$.

Figure 6 illustrates the effect of interparticle collisions on the distributions of $q_{\mathrm{w}}$ and $e_{\mathrm{w}}$ over the whole forward part of the cylinder. For small particles $\left(r_{\mathrm{p}}=0.5 \mu \mathrm{m}\right)$, the collisions essentially influence the heat flux $q_{\mathrm{w}}$ in the vicinity of the stagnation point up to $\theta=\pi / 4$, whereas they have almost no effect at 


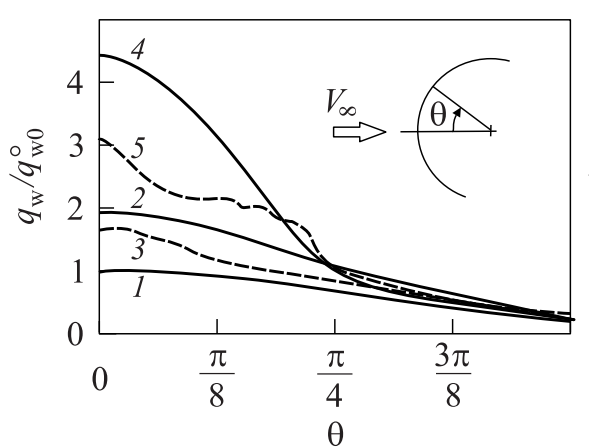

(a)

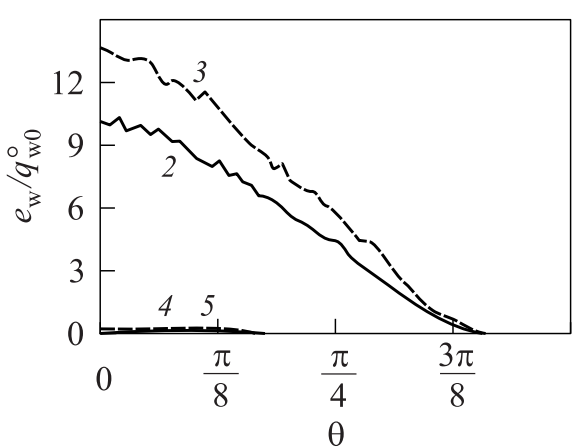

(b)

Figure 6 Distributions of the heat flux from the carrier gas $q_{\mathrm{w}}$ and the energy flux from the dispersed phase $e_{\mathrm{w}}$ along the surface of the cylinder for various particle radii: $1-\alpha_{\mathrm{p} \infty}=0$ (dust-free gas flow); and $2-5-\alpha_{\mathrm{p} \infty}=10^{-5}\left(2\right.$ and $3-r_{\mathrm{p}}=1 \mu \mathrm{m}$; 4 and $5-0.5 \mu \mathrm{m} ; 2$ and 4 - with interparticle collisions; and 3 and 5 - without interparticle collisions)

$\theta \geq \pi / 4$. The effect of the collisions of such particles on $e_{\mathrm{w}}$ is very weak. The collisions of larger particles $\left(r_{\mathrm{p}}=1 \mu \mathrm{m}\right)$, on the contrary, noticeably affect the particle energy flux to the body surface, resulting in $30 \%-40 \%$ reduction in $e_{\mathrm{w}}$. This phenomenon is known as the shielding effect. Note that fine particles $\left(r_{\mathrm{p}}<r_{\mathrm{p} *}\right)$ move in the flow without collisions.

Both the heat flux $q_{\mathrm{w}}$ and the energy flux $e_{\mathrm{w}}$ increase with the free stream particle volume fraction $\alpha_{\mathrm{p} \infty}$ (Fig. $7 a$ ). The rate of increase essentially depends

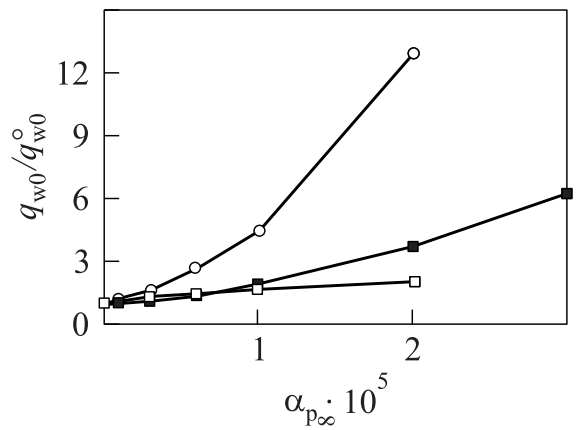

(a)

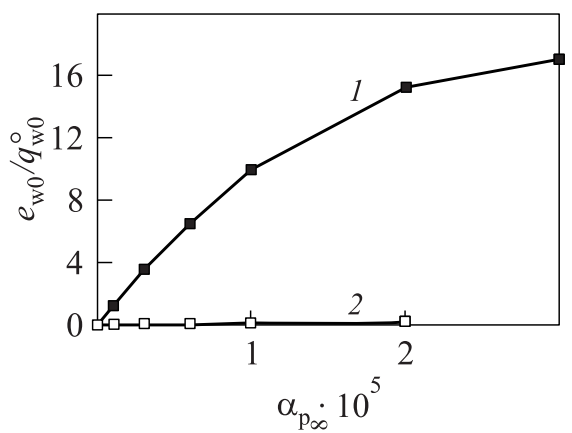

(b)

Figure 7 Plots of (a) heat flux from the carrier gas $q_{\mathrm{w} 0}$; and $(b)$ energy flux from the dispersed phase $e_{\mathrm{w} 0}$ to the cylinder surface at the stagnation point vs. the free stream particle volume fraction $\alpha_{\mathrm{p} \infty}: 1-r_{\mathrm{p}}=1 \mu \mathrm{m} ; 2-0.5$; and $3-r_{\mathrm{p}}=0.3 \mu \mathrm{m}$. Signs are the calculated points 
on the particle radius. For particles whose radius is larger than $r_{\mathrm{p} *}$, the dependence $q_{\mathrm{w}}\left(\alpha_{\mathrm{p} \infty}\right)$ is nonlinear (see Fig. 7a). The dependence $e_{\mathrm{w} 0}\left(\alpha_{\mathrm{p} \infty}\right)$ for larger particles $\left(r_{\mathrm{p}}=1 \mu \mathrm{m}\right)$ is also nonlinear, but contrary to $q_{\mathrm{w}}\left(\alpha_{\mathrm{p} \infty}\right)$, the plot of $e_{\mathrm{w} 0}\left(\alpha_{\mathrm{p} \infty}\right)$ seems to tend to the maximum with increasing $\alpha_{\mathrm{p} \infty}$ (see Fig. $\left.7 b\right)$. The similar effect was described in [7].

\subsection{Estimation of Contact Heat Transfer in Particle-Wall Collisions}

The heat flux from the dispersed phase to the body surface $q_{c}$ due to the contact heat transfer during particle-wall collisions was estimated with the use of the relation proposed in [13], and it was found to be much less than $q_{\mathrm{w}}+e_{\mathrm{w}}$ in the whole range of particle radii considered in the study.

\subsection{Estimation of Turbulence Generation by Particles}

The carrier gas flow was computed assuming that velocity fluctuations are negligible. Due to their inertia, particles exhibit very large relative velocities just behind the shock wave; therefore, the particle Reynolds number in this region may be high enough for the turbulence generation to be significant.

In order to estimate the level of the turbulence arising from this phenomenon, the turbulent kinetic energy $k$ along the stagnation streamline from the shock wave to the body surface was calculated considering the computed gas flow parameters as the average ones and using the Crowe approach [14] with some modifications. Then the average fluctuating velocity of the carrier gas $w^{\prime}=\sqrt{2 k / 3}$ was calculated. The ratio $w^{\prime} / V_{\infty}$ represents the relative turbulence intensity. At fixed particle volume fraction $\alpha_{\mathrm{p} \infty}$, the level of $w^{\prime} / V_{\infty}$ just behind the bow shock is higher for small particles than for large ones. For example, at $\alpha_{\mathrm{p} \infty}=10^{-5}$, the estimated value of $w^{\prime} / V_{\infty}$ for $r_{\mathrm{p}}=0.3 \mu \mathrm{m}$ (this value is less than $r_{\mathrm{p} *}$ ) is approximately equal to 0.018 , whereas for $r_{\mathrm{p}}=3 \mu \mathrm{m}$, it is equal to 0.013 . However, the turbulence intensity increases towards the body surface in the case of larger particles and considerably decreases if particles are fine $\left(r_{\mathrm{p}} \leq r_{\mathrm{p} *}\right)$.

Distributions of $w^{\prime} / V_{\infty}$ along the stagnation streamline near the surface of a cylinder are displayed in Fig. 8 for various $r_{\mathrm{p}}$ at $\alpha_{\mathrm{p} \infty}=10^{-5}$. We compared these levels of $w^{\prime}$ at the outer boundary of the boundary layer with the levels of particles' fluctuating velocities $w_{\mathrm{p}}^{\prime}$ due to the interparticle collisions and found that $w^{\prime}$ is several times less than $w_{\mathrm{p}}^{\prime}$ for particles of radii $r_{\mathrm{p}} \geq 1 \mu \mathrm{m}$, these fluctuating velocities are of the same order for particles of radii close to $r_{\mathrm{p} *}$, and $w_{\mathrm{p}}^{\prime}$ is much less than $w^{\prime}$ for very fine particles $\left(r_{\mathrm{p}} \leq 0.3 \mu \mathrm{m}\right)$. In the latter case, the level of $w^{\prime}$ itself is very low. Therefore, the turbulence generated by particles plays a negligible role in the modification of the behavior of large particles near the body surface, while it can play a noticeable role if the particle radius is in the vicinity of $r_{\mathrm{p} *}$. On the other hand, as is known, the turbulence of the carrier gas 


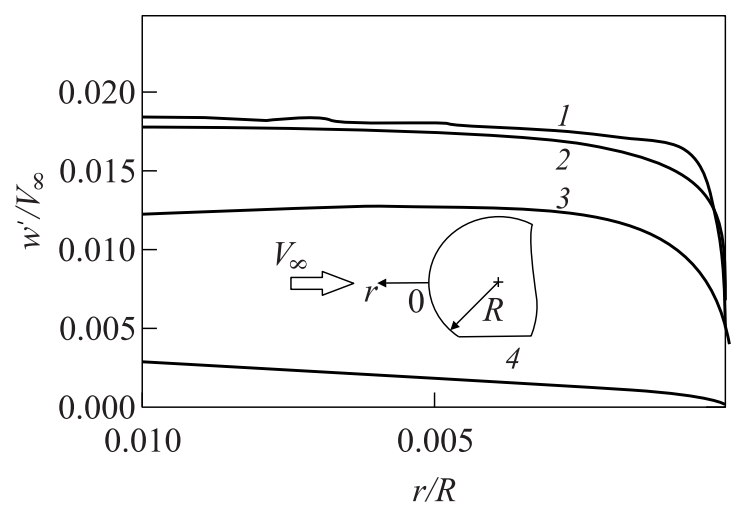

Figure 8 Turbulence intensity of the carrier gas $w^{\prime} / V_{\infty}$ caused by particles vs. the distance from the surface of the cylinder along the stagnation streamline; $\alpha_{\mathrm{p} \infty}=10^{-5}$ : $1-r_{\mathrm{p}}=3 \mu \mathrm{m} ; 2-1 ; 3-0.5 ;$ and $4-r_{\mathrm{p}}=0.3 \mu \mathrm{m}$

at the outer boundary of the boundary layer can increase considerably the heat flux at the stagnation point $q_{\mathrm{w} 0}$. A simple estimation of this effect based on the empirical formulae [15] shows that the levels of $w^{\prime} / V_{\infty}$ given in Fig. 8 are high enough to increase the magnitude of $q_{\mathrm{w} 0}$ from several percents in the case of fine particles $\left(r_{\mathrm{p}}=0.3 \mu \mathrm{m}\right)$ to several tens of percents in the case of $r_{\mathrm{p}}=0.5 \mu \mathrm{m}$ that is close to $r_{\mathrm{p} *}=0.45 \cdot 10^{-4} \mu \mathrm{m}$. For large particles, the turbulence modulation has a negligible effect on the total heat flux $q_{\mathrm{w} 0}+e_{\mathrm{w} 0}$ because $e_{\mathrm{w} 0} \gg q_{\mathrm{w} 0}$ and $e_{\mathrm{w} 0}$ practically does not depend on $w^{\prime}$ under the considered flow conditions. To conclude we may say that both the particle behavior and the heat transfer in the stagnation region are rather sensitive to the turbulent modulation by particles if the particle radius is close to the critical value $r_{\mathrm{p} *}$. Hence, a further model development for high speed two-phase flow over a blunt body requires this modulation to be taken into account, and this must be a subject of investigations in the future.

\section{ACKNOWLEDGMENTS}

The most part of the research reported in this paper has been carried out thanks to financial support from the INTAS through grant No.00-0309. The last two authors wish also to acknowledge the partial support of the study from the Russian Foundation for Basic Research (grant No. 02-01-01201) and the Ministry of Education of Russia (grant No. E02-4.0-138). 


\section{REFERENCES}

1. Dunbar, L. E., J.F. Courtney, and L. D. McMillen. 1975. Heating augmentation in erosive hypersonic environments. AIAA J. 13(7):908-12.

2. Hove, D. T., and E. Taylor. 1976. Stagnation region heat transfer in hypersonic particle environments. AIAA J. 10:1486-88.

3. Vasilevskii, E. B., L. A. Dombrovskii, D. S. Mikhatulin, and Yu. V. Polezhaev. 2001. Heat transfer in the neighborhood of the stagnation point under conditions of hypersonic heterogeneous slip flow past bodies. High Temperature 39(6):860-73.

4. Osiptsov, A. N., L. A. Egorova, V. I. Sakharov, and Boyi Wang. 2002. Heat transfer in supersonic dusty-gas flow past a blunt body with inertial particle deposition effect. Progr. Natural Sci. 12(12):887-92.

5. Osiptsov, A.N., and E. G. Shapiro. 1986. Effect of fine particles on the boundary layer structure in hypersonic flow past a blunt body. Izv. Akad. Nauk SSSR, Mekh. Zhidk. Gasa 5:55-62. [In Russian.]

6. Vasilevskii, E. B., A. N. Osiptsov, A. V. Chirikhin, and L. V. Yakovleva. 2001. Heat transfer at the forward surface of a blunt body in a high speed flow containing low-inertial particles. Inzhenerno-Fizicheskii Zhurnal 74(6):29-37. [In Russian.]

7. Kitron, A., T. Elperin, and A. Tamir. 1989. Monte Carlo analysis of wall erosion and direct contact heat transfer by impinging two-phase jet. J. Thermophys. Heat Transfer 3(2):112-22.

8. Tsirkunov, Yu. M. 2001. Gas-particle flows around bodies - key problems, modeling and numerical analysis. 4th Conference (International) on Multiphase Flow. CD-ROM. Paper No.607. 31 p..

9. Volkov, A. N., and Yu. M. Tsirkunov. 2000. Kinetic model of collisional admixture in dusty gas and its application to calculating flow past bodies. Fluid Dynamics 35(3):380-92.

10. Volkov, A.N., and Yu. M. Tsirkunov. 2000. Computational simulation of viscous two-phase flows of a dense gas-particle mixture over bodies. ECCOMAS'2000 Proceedings. CD-ROM. Paper No. 309. 20 p.

11. Volkov, A. N., and Yu. M. Tsirkunov. 2002. Numerical investigation of the shock layer structure and heat transfer at the surface of a body in a supersonic dusty gas flow. 5th World Congress on Computational Mechanics Proceedings. Paper No. 80339. 11 p..

12. Volkov, A.N., and Yu. M. Tsirkunov. CFD / Monte Carlo simulation of collisiondominated gas-particle flows over bodies. ASME FEDSM'2002 Proceedings. Paper No. 31222. 14 p.

13. Sun, J., and M. M. Chen. 1988. A theoretical analysis of heat transfer due to particle impact. Int. J. Heat Mass Transfer 31:969-75.

14. Crowe, C. T. 2000. On models for turbulence modulation in fluid-particle flows. Int. J. Multiphase Flow 26:719-27.

15. Gorshkov, G. F. 1992. Heat transfer in impact jet systems under the strong effect of initial perturbations. Dr.Sci. Thesis. St. Petersburg Mechanical Institute, St. Petersburg. 